345-352

William ECHARD. Psychedelic Popular Music: A History Through Musical Topic Theory. Indiana University Press, 2017, 306 pp.

El concepto de tópico musical fue acuñado por Leonard Ratner en la década de los 80 en su libro Classic Music: Expression, Form, and Style. Esta primera aproximación sugirió un cambio notable que se observaría luego en la semiología de la música, en concreto, en el estudio de las prácticas musicales del clasicismo y romanticismo; - un ejemplo claro de esto es el The Oxford Handbook of Topic Theory; editado por Danuta Mirka y publicado en 2014 por la editorial Oxford University Press.

La delimitación del concepto de tópico musical resulta ser compleja, incluso en la actualidad; no obstante, se ha transformado en uno de los puntos de mayor interés para los interesados de la semiótica de la música. A rasgos generales, una de las definiciones más masificadas es la del musicólogo Ghanés Kofi Agawu, el que lo definió como un signo, que posee un significante -asociado a dimensiones melódicas, rítmicas y armónicas-correlacionado con un significado. Por otra parte, el investigador mexicano Rubén López-Cano, la teoría de los tópicos ha permeado de manera significativa las orientaciones investigativas en el campo musicológico de los últimos años, por tanto, es común advertir una creciente inclinación por la aplicación de este enfoque en contextos diversos. Así, William Echard quien es profesor asociado de la Universidad de Carleton, Ottawa, Canadá, señala que el estudio de la música psicodélica a partir de dicho modelo teórico, supone un ámbito fecundo que permite determinar cómo manifestaciones diversas confluyen en un espacio conjunto de comprensión. La producción científica del autor se relaciona con la indagación de la música popular urbana desde una perspectiva semiológica. Se centra en una semiótica social, asociada en particular al proceso cultural de recepción de la obra. En esta línea de trabajo se puede observar su libro Psychedelic Popular Music: A History Through Musical Topic Theory, donde explora el devenir histórico de las expresiones asociadas al género psicodélico y sus implicaciones a partir de la década de los 60 hasta la fecha.

La estructura del libro podría interpretarse en dos momentos. En primer lugar, el texto expone un estudio que fundamenta el concepto de psicodelia aplicado a la música popular, mientras que los siguientes capítulos reportan una suerte de historia de dichas manifestaciones psicodélicas a partir de la teoría de tópicos -el mismo autor señala que no es una investigación a cabalidad de todos los aspectos históricos y disciplinarios de aquel género; sin embargo, es un relato centrado en las características tópicas de aquella expresión.

En el capítulo inicial, denominado Delineating Psychedelia: Topic Theory and Popular Music Cultures, el autor delimita las relaciones existentes entre la teoría de tópicos musicales y la música catalogada dentro del género psicodélico. Aquel ejercicio resulta ser de interés, por cuanto Echard propone la transferibilidad de un modelo que ha sido comúnmente utilizado en el estudio la música escrita de tradición occidental. 
Estas definiciones preliminares le otorgan al lector las concepciones básicas para comprender las secciones siguientes del texto, lo que se expone de manera clara y da sentido al primer momento de este libro. Es interesante observar en esta sección las determinaciones epistemológicas que sustentan la visión de la teoría de tópicos por parte del investigador. Esto último permite establecer las bases con las que el relato histórico se entrelaza con las reflexiones semióticas que surgen a propósito del análisis de algunos casos emblemáticos en la historia de dicha estética

En el segundo capítulo, Developments Through 1966, el autor expone las principales cuestiones asociadas al desarrollo del rock psicodélico por medio del análisis de las cualidades tópicas de The Yardbirds. Un aspecto relevante a considerar tiene relación con las características idiomáticas de la época que son observadas por Echard en esa agrupación. De esta forma, menciona que las etapas posteriores de dicho periodo se configuran por medio de los tópicos musicales que surgen en su producción creativa. A propósito de dicha banda, el investigador realiza una revisión de las bases más significativas en cuanto a contenidos que son evidenciables a partir de la pesquisa de las canciones Heart Full of Soul y Happening Ten Years Ago. De manera posterior, el académico profundiza en otro grupo contemporáneo al anterior, The Kinks; quienes desde su perspectiva conforman uno de los ejemplos representativos de la psicodelia temprana, influenciada mediante una adaptación de ciertos elementos de la música clásica india -con procedimientos semejantes a los observados en otros tipos de orientalismos aplicados en Occidente-. Así, se recurre al estudio de la canción See My Friends, donde se aíslan determinados patrones que permiten visibilizar la presencia de aspectos propios de los orientalismos y exotismos señalados antes. Asimismo, reporta la existencia de distintos componentes que remiten a ciertas prácticas propias de la monodia sacra medieval. Mas adelante analiza las obras Eight Miles High de The Byrds, hasta llegar a su primera alusión a los Beatles. El apartado concluye indicando que los inicios de la década de los 60 son un campo tópico fecundo para la configuración de las partes que se transformarán en ejes característicos de la estética psicodélica. Este marco referencial, señalado por el autor, permite la resignificación de algunos tópicos preexistentes y también posibilita el surgimiento de nuevos, como el Indio, gregoriano, folclórico, belle époque, circo, espacio y jazz -en variadas formas-.

El siguiente capítulo, llamado The Later 1960s, adquiere una estructura diferente. En este sentido, el investigador menciona que los dos primeros apartados tuvieron por objetivo introducir los tópicos musicales centrales en esa estética. Esto permite que la sección se centre en aquellos aspectos sustanciales que determinan una linealidad en cuanto su emergencia. En particular, el autor comienza con una revisión del Soul y el Funk en el contexto de la psicodelia temprana -entendida como la música psicodélica de la década del 60-. De manera posterior, es posible observar una segunda aproximación de los Beatles, y los análisis iniciales de la música de Pink Floyd. Acerca de lo último, Echard reporta las características musicales que transforman al trabajo temprano de dicha agrupación en un ícono de la psicodelia de finales de los 60. Asimismo, identifica la 
existencia de rasgos característicos de la banda y también el surgimiento de los tópicos musicales que fueron mencionados antes, lo que se observa en particular en el uso de órgano eléctrico, lo rural y lo pastoral, la tendencia a generar esquemas rítmico-melódicos de propiedades reiterativas y de carácter improvisado.

El cuarto capítulo se denomina The 1970s, década que es planteada por el investigador a modo de periodo de transición. Se caracteriza por una presencia sustancial de temáticas asociadas al espacio, en lo que Echard llama rock espacial (space rock). La categoría emerge para transformarse en un elemento distintivo a partir finales de los $60 \mathrm{y}$ Pink Floyd es la banda que logra identificarla. En particular, el autor analiza la canción de Astronomy Domine, de la agrupación ya señalada. Dicha obra es catalogada en el texto como un trabajo directamente relacionado con la dimensión tópica del espacio. Otro músico estudiado es Jimy Hendrix, mediante Third Stone From The Sun, que presenta algunas particularidades a diferencia del caso de Pink Floyd. En tal sentido, en el libro se reportan los nexos utilizados por Hendrix para transitar desde alusiones al espacio, a una concepción ideológica y espiritual de dicho asunto. El apartado finaliza con un recuento de la transformación del tópico espacial, hacia representaciones cada vez más comunes -sobre todo en el rock psicodélico-.

El capítulo final, denominado The 1980s and On presenta los aspectos más significativos del estado actual de la música psicodélica, a partir de un giro estético en la década de los 80, hasta el britpop de los 90. Echard menciona que los tópicos identificados en años anteriores muestran la emergencia de nuevos estilos y combinaciones musicales que expresan las cualidades contenidas en aquellos elementos tópicos reportados con anterioridad. Desde el punto de vista analítico, el autor reporta análisis de The Soft Boys por medio de la canción Underwater moonlight; The Butthole Suffers, con Creep in The Cellar (y algunas menciones a las canciones Sea Ferring y Strangers Die Every Day); Primal Scream, con Loaded; The Orbs, con Little Fluffy Clouds; y The Shamen, por medio de Revolution y Ebeneezer Goode.

A partir de lo señalado, es posible advertir una vasta revisión de repertorio musical asociado a la música psicodélica. La determinación de los tópicos tratados es consistente a lo largo de la obra y se evidencian en numerosos ejemplos musicales. Asimismo, el autor reporta que dicho texto representa más bien una aproximación poco exhaustiva, pero contribuye a una tarea fundamental en la ciencia: probar la transferibilidad de conceptos, ideas y categorías. Al respecto, Echard menciona que el ejercicio intelectual realizado a propósito de la escritura del libro aquí reseñado, permitió evidenciar la existencia de nuevos campos tópicos y también de tópicos musicales.

En definitiva, el texto de Echard aporta una interesante mirada al desarrollo de la música popular, a partir de un enfoque semiológico. Si bien esta perspectiva de investigación ha comenzado a instalarse con fuerza en investigadores jóvenes, pueden ser sustanciales los aportes de estas miradas a la configuración de una dimensión compleja del fenómeno musical urbano. De tal forma, y como lo reporta el mismo autor, los avances asociados al 
estudio de la música popular, su componente analítico social y crítico contribuyen de manera significativa al enriquecimiento de la teoría de tópicos musicales.

Esta reseña forma parte de la revisión bibliográfica realizada en el marco del proyecto Fondecyt 11190505 financiado por ANID, Chile.

https://doi.org/10.32735/S0718-2201202000050800

Ignacio Soto Silva

Universidad de los Lagos (Chile)

ignacio.soto@ulagos.cl

\section{Virginia SÁNCHEZ RODRíGUEZ. La soprano María Barrientos y sus epístolas de juventud (1905-1906). Málaga: Universidad de Málaga, 2018, 198 pp.}

De un tiempo a esta parte, siguen surgiendo una gran cantidad de estudios científicos de notoria calidad dedicados a la recuperación y el análisis de la vida y obra de un buen número de mujeres que destacaron en los campos de la ciencia o el arte. Así, por distintos motivos, estas parecían haber sido olvidadas o relegadas a un segundo plano, una omisión que, tristemente, se ha perpetuado con el paso de los años.

En el campo de las artes en general y de la música en particular, la hegemonía masculina ha sido una realidad a lo largo de los siglos. Si nos aproximamos a la historia de la música y a la investigación musical, en concreto, la preponderancia del patriarcado es más que evidente, ya que son mayoritarias las referencias que se han dedicado a los compositores (también a intérpretes y musicólogos, aunque en menor medida). Por su parte, las mujeres han sido arrinconadas por la historiografía pese a que no pocas lograron destacar en diferentes ámbitos: incluso, en muchos casos, fueron reconocidas y aplaudidas por sus contemporáneos. De forma errónea, hay quien señala que esta falta se debe a la ausencia de participación o la carencia de interés de las féminas en estas tareas. Por fortuna, actualmente, como mencionamos, al breve listado ya conocido se van sumando numerosos nombres de ejecutantes, creadoras, investigadoras y mecenas que realizaron una labor relevante en estos aspectos.

Si nos centramos en el campo de la ópera, las mujeres han podido desarrollarse profesionalmente y trazar trayectorias profesionales muy destacadas. En muchas ocasiones, se circunscribían a roles específicos muy delimitados que se emplazaban en las convenciones sociales y contextos laborales preponderantes en cada momento histórico.

Es el caso que nos ocupa, Virginia Sánchez Rodríguez publicó en 2018 un excelente y recomendable trabajo de María Barrientos (1884-1946). La soprano española, internacionalmente conocida en su momento, desarrolló su carrera dentro de un terreno dominado y gobernado por los hombres. Considerada una diva, cantó en los principales escenarios de América Latina, Europa y América del Norte, aunque, hasta este momento, no había recibido la atención que, sin duda, merece, paliada en buena parte por esta investigación.

Respecto de la autora, es Licenciada en Historia del Arte y ha cursado los másteres en Música Hispana y Formación del Profesorado. Experta en Gestión Cultural 